\title{
POINT EVALUATION AND HARDY SPACE ON A HOMOGENEOUS TREE
}

\author{
DANIEL ALPAY AND DAN VOLOK
}

\begin{abstract}
We consider stationary multiscale systems as defined by Basseville, Benveniste, Nikoukhah and Willsky. We show that there are deep analogies with the discrete time non stationary setting as developed by the first author, Dewilde and Dym. Following these analogies we define a point evaluation with values in a $C^{*}$-algebra and the corresponding "Hardy space" in which Cauchy's formula holds. This point evaluation is used to define in this context the counterpart of classical notions such as Blaschke factors.
\end{abstract}

\section{INTRODUCTION}

The purpose of this paper is to explain relationships between the theory of nonstationary linear systems indexed by the integers and the theory of stationary linear systems indexed by a homogeneous tree. We restrict ourselves to the case of input/ouput systems and postpone the treatment of state space realizations to a future publication. Our motivation for this study originates with the works of Basseville, Benveniste, Nikoukhah and Willsky (see [12, 11, 10, 9]) where a general theory of multiscale system is developed. In particular these authors distinguished special families of stochastic processes (stationary and isotropic) and developed Schur-Levinson recursions for isotropic processes. They also distinguish a special family of operators which they call stationary transfer functions. We associate to such functions point evaluations whose domain and range are in a $C^{*}$-algebra associated to the tree. We explain the relationships with the point evaluation defined for upper-triangular operators in 22. The analogies between the two cases allow interfeeding. In particular, one can pose and solve interpolation problems which should have applications to the theory of multiscale systems. We plan to consider this in a future publication. Some of the results presented here were announced in [4.

The outline of the paper is as follows. In Section 2 we briefly review the setting developed in 22. In the third Section we present part of the multiscale system theory relevant to the present study. Although in part of a review nature, the point of view contains some novelties, in particular by considering the $\ell_{2}$ space associated to the tree. An important role is played by the so-called Cuntz relations (see [16]). In the fourth Section we introduce the $C^{*}$-algebra of constants and the Hardy space associated to the tree. In Section 5 we study the properties of the point evaluation

1991 Mathematics Subject Classification. Primary: 93B28; Secondary: 05 C05.

Key words and phrases. System realization, homogeneous tree, Hilbert module.

The first author was supported by the Israel Science Foundation (Grant no. 322/00). 
and the notion of Schur multipliers is studied in Section [6

We would like to mention that similar techniques were used by Constantinescu and Johnson (see [14, 15]) in a different setting (tensor algebras rather than Cuntz algebras). Elements of system theory for Cuntz algebras are being considered by Ball and Vinnikov in [8] and [7. Also, the connections between Cuntz algebras and wavelets are studied in the book 22.

Acknowledgments: It is a pleasure to thank Prof. J. Ball for insightful comments on preliminary versions of this paper, and Prof. A. Benveniste who made available to us the reports [9] and 11], which sparked our interest in the subject.

\section{THE DISCRETE TIME NON STATIONARY SETTING}

We briefly review the nonstationary setting developped in [1] and [2]. We fix a separable Hilbert space $\mathcal{N}$, the coefficient space, and consider the Hilbert space $\ell_{\mathcal{N}}^{2}$ of all two sided square summable sequences $f=(f)_{i=-\infty}^{\infty}=\left(\ldots, f_{-1}, f_{0}, f_{1}, \ldots\right)$ with components $f_{i} \in \mathcal{N}$ provided with the standard inner product. The set of bounded linear operators from $\ell_{\mathcal{N}}^{2}$ into itself is denoted by $\mathcal{X}$. Let $Z$ denote the bilateral backward shift operator

$$
(Z f)_{i}=f_{i+1}, \quad i=\ldots,-1,0,1, \ldots
$$

It is unitary on $\ell_{\mathcal{N}}^{2}$. An element $A \in \mathcal{X}$ can be represented as an operator matrix $\left(A_{i j}\right)$ with $A_{i j}=\pi^{*} Z^{i} A Z^{* j} \pi$ where $\pi$ denotes the injection map: $u \in \mathcal{N} \mapsto$ $(\ldots, 0, u, 0, \ldots) \in \ell_{\mathcal{N}}^{2}$. We denote by $\mathcal{U}$ and $\mathcal{D}$ the spaces of upper triangular and diagonal operators :

$$
\mathcal{U}=\left\{A \in \mathcal{X}: A_{i j}=0, i>j\right\}, \quad \mathcal{D}=\left\{A \in \mathcal{X}: A_{i j}=0, i \neq j\right\} .
$$

For $W \in \mathcal{D}$ we set $W^{(j)}=Z^{* j} W Z^{j}$ and

$$
W^{[0]}=I, \quad W^{[n]}=W W^{(1)} W^{(2)} \ldots W^{(n-1)}=\left(W Z^{*}\right)^{n} Z^{n}, \quad n \geq 1 .
$$

Theorem 2.1. Let $F \in \mathcal{U}$ and $D \in \mathcal{D}$. There exists a unique sequence of operators $F_{[j]} \in \mathcal{D}, j=0,1, \ldots$, namely $\left(F_{[j]}\right)_{i i}=F_{i-j, i}$, such that

$$
F=\sum_{n=0}^{\infty} Z^{n} F_{[n]}
$$

in the sense that $F-\sum_{j=0}^{n-1} Z^{j} F_{[j]} \in Z^{n} \mathcal{U}$. The operator $(Z-W)^{-1}(F-D)$ belongs to $\mathcal{U}$ for $W \in \mathcal{D}$ such that $r_{s p}\left(Z^{*} W\right)<1$ if and only if

$$
D=\sum_{n=0}^{\infty} W^{[n]} F_{[n]} \stackrel{\text { def. }}{=} F^{\wedge}(W) .
$$

An operator $F=\left(F_{i j}\right) \in \mathcal{X}$ is a Hilbert-Schmidt operator if all its entries $F_{i j}$ are Hilbert-Schmidt operators on $\mathcal{N}$ and $\sum_{i j} \operatorname{Tr} F_{i j}^{*} F_{i j}<\infty$, where $\operatorname{Tr}$ stands for trace. The set of these operators will be denoted by $\mathcal{X}_{2}$ and it is a Hilbert space with respect to the inner product

$$
\langle F, G\rangle_{\mathcal{X}_{2}}=\sum_{i j} \operatorname{Tr} G_{i j}^{*} F_{i j}<\infty .
$$


The subspaces of upper triangular and diagonal operators which are moreover Hilbert-Schmidt operators on $\mathcal{N}$ will be denoted by $\mathcal{U}_{2}$ and $\mathcal{D}_{2}$. The space $\mathcal{U}_{2}$ is a reproducing kernel Hilbert space with reproducing kernel

$$
\rho_{W}^{-1}=\left(I-Z W^{*}\right)^{-1}=\sum_{0}^{\infty}\left(Z W^{*}\right)^{n}=\sum_{0}^{\infty} Z^{n} W^{[n] *}
$$

in the sense that for all $W \in \Omega, E \in \mathcal{D}_{2}$, and $F \in \mathcal{U}_{2}$, the operator $\rho_{W}^{-1} E \in \mathcal{U}_{2}$ and

$$
\left\langle F, \rho_{W}^{-1} E\right\rangle_{\mathcal{U}_{2}}=\operatorname{Tr} E^{*} F^{\wedge}(W) .
$$

This last formula is the non stationary counterpart of Cauchy's formula for Hardy functions.

The map $W \mapsto F^{\wedge}(W)$ (which was first introduced in 1]) and its counterpart when one replaces $(Z-W)^{-1}(F-D)$ by $(F-D)(Z-D)^{-1}$ in the above theorem allow to translate most, if not all, of the classical analysis of the Hardy space $H_{2}$ to the setting of upper triangular operators. The analogue of the Hardy space $H_{2}$ is given by the Hilbert space of upper triangular operators $\mathcal{U}_{2}$. See [3], 6], 17 for sample applications. As already mentioned and as we will see in the sequel, they have analogues in the setting of multiscale system theory.

\section{Multiscale System theory}

Some of the fundamental notions in the classical theory of discrete time linear systems are that of causality and stationarity. In this section we review the analogues of these notions, introduced by Basseville, Benveniste, Nikoukhah and Willsky in the case of multiscale linear systems.

Let $\mathcal{T}$ be a homogeneous tree of order $q \geq 2$ - an infinite acyclic, undirected, connected graph such that every node has exactly $q+1$ branches (see 24, 13]). We consider a linear system of the form

$$
g(t)=(S f)(t),
$$

where the input signal $f=f(t)$ and the output signal $g=g(t)$ belong to the Hilbert space $\ell_{2}(\mathcal{T})$ of square-summable sequences, indexed by the nodes of $\mathcal{T}$, and where $S$ is a bounded linear operator on $\ell_{2}(\mathcal{T})$ (notation: $S \in \mathbf{X}(\mathcal{T})$ ). Using the notation $\chi_{t}$ for the element of the standard basis of $\ell_{2}(\mathcal{T})$, supported at the node $t$, one can write

$$
(S f)(t)=\sum_{u \in \mathcal{T}} s_{t, u} f(u), \text { where } s_{t, u}=\left[S \chi_{u}, \chi_{t}\right] \in \mathbb{C}
$$

and where the sum is absolutely convergent by Cauchy-Schwarz inequality.

According to the standard tree compactification procedure, a boundary point of $\mathcal{T}$ is an equivalence class of infinite paths modulo finite number of edges. Let us distinguish a boundary point of $\mathcal{T}$ and denote it by $\infty_{\mathcal{T}}$. Then for each $t \in \mathcal{T}$ there exists a unique representative $\mathcal{T}_{t}^{-}$of the equivalence class $\infty_{\mathcal{T}}$, starting at $t$. For a pair of nodes $t, s$, the paths $\mathcal{T}_{t}^{-}, \mathcal{T}_{s}^{-}$have to coincide after a finite number of edges; the first of their common nodes is denoted by $s \wedge t$. The notion of distance $\operatorname{dist}(s, t)$ 
between the nodes $s, t$, defined as the number of edges along the path connecting $s$ and $t$, allows to introduce the partial order

$$
s \preceq t \quad \text { if } \quad \operatorname{dist}(s, s \wedge t) \leq \operatorname{dist}(t, s \wedge t)
$$

and the equivalence relation

$$
s \asymp t \quad \text { if } \quad \operatorname{dist}(s, s \wedge t)=\operatorname{dist}(t, s \wedge t) .
$$

The equivalence classes, defined with respect to the equivalence relation above, are called horocycles.

Definition 3.1. The multiscale linear system (3.1) is said to be causal if for every node $t \in \mathcal{T}$ the subspace

$$
\left\{f \in \ell_{2}(\mathcal{T}): t \preceq \operatorname{support}(f)\right\}
$$

is $S$-invariant.

In order to analyze Definition 3.1 we consider the primitive shifts on the tree. By convention they act on the right and are defined as follows. The primitive upward shift $\bar{\gamma}: \mathcal{T} \mapsto \mathcal{T}$ is determined by

$$
\forall t \in \mathcal{T}: t \bar{\gamma} \preceq t, \operatorname{dist}(t \bar{\gamma}, t)=1 .
$$

In the choice of the primitive downward shifts there is some freedom; we assume that some such choice $\alpha_{i}: \mathcal{T} \mapsto \mathcal{T}, 1 \leq i \leq q$,

$$
\forall t \in \mathcal{T}:\{s \in \mathcal{T}: t \preceq s, \operatorname{dist}(t, s)=1\}=\left\{t \alpha_{1}, \ldots, t \alpha_{q}\right\},
$$

is fixed, as well. Furthermore we consider the primitive shift operators, acting on the left on $\ell_{2}(\mathcal{T})$ and defined via convolution:

$$
\bar{\gamma} f(t)=\frac{1}{\sqrt{q}} f(t \bar{\gamma}), \alpha_{i} f(t)=f\left(t \alpha_{i}\right) .
$$

We compute the adjoint operators $\gamma=\bar{\gamma}^{*}, \bar{\alpha}_{i}=\alpha_{i}^{*}$ :

$$
\gamma f(t)=\frac{1}{\sqrt{q}} \sum_{s \bar{\gamma}=t} f(s), \bar{\alpha}_{i} f(t)= \begin{cases}f(t \bar{\gamma}), & t=t \bar{\gamma} \alpha_{i}, \\ 0, & \text { otherwise },\end{cases}
$$

and observe that the following relations hold true:

$$
\begin{gathered}
\alpha_{i} \bar{\alpha}_{j}=\delta_{i, j}, \quad \sum_{i=1}^{q} \bar{\alpha}_{i} \alpha_{i}=1, \\
\bar{\gamma}=\frac{1}{\sqrt{q}} \sum_{i=1}^{q} \bar{\alpha}_{i}, \quad \gamma \bar{\gamma}=1 .
\end{gathered}
$$

Equations (3.3) are called the Cuntz relations. Equation (3.4) implies that the primitive upward shift operator $\bar{\gamma}$ is an isometry from $\ell_{2}(\mathcal{T})$ into itself. However, it is not surjective and thus $\bar{\gamma}$ is not unitary (see also (4.2) and (4.3) below). We also observe that for any pair of nodes $t, u \in \mathcal{T}$ there exist a unique choice of indices $i_{1}, \ldots, i_{n}, j_{1}, \ldots, j_{m}$, such that

$$
t=(t \wedge u) \alpha_{i_{n}} \cdots \alpha_{i_{1}}, u=(t \wedge u) \alpha_{j_{m}} \cdots \alpha_{j_{1}}
$$

(note that, according to the definition of $t \wedge u, j_{m} \neq i_{n}$ ). Then for any $f \in \ell_{2}(\mathcal{T})$ it holds that

$$
f(u)=\bar{\alpha}_{i_{1}} \cdots \bar{\alpha}_{i_{n}} \alpha_{j_{m}} \cdots \alpha_{j_{1}} f(t) .
$$


This observation leads to a multiscale analogue of Theorem 2.1 It can be formulated in terms of the point-wise convergence of a sequence of bounded operators on $\ell_{2}(\mathcal{T})$ : we shall say that a sequence of bounded operators $S_{n}$ converges point-wise to a bounded operator $S$ if for every $f \in \ell_{2}(\mathcal{T})$ and $t \in \mathcal{T} \lim _{n \rightarrow \infty}\left(S_{n} f\right)(t)=(S f)(t)$. We note that on the dense subspace of finitely supported functions the point-wise convergence implies the convergence in strong operator topology.

Theorem 3.2. Any operator $S \in \mathbf{X}(\mathcal{T})$ can be represented as the point-wise converging series

$$
S=\sum_{n, m=0}^{\infty} \sum_{\substack{i_{1}, \ldots, i_{n} \leq q \\ j_{1}, \ldots, j_{m} \\ j_{m} \neq i_{n}}} \bar{\alpha}_{i_{1}} \cdots \bar{\alpha}_{i_{n}} \alpha_{j_{m}} \cdots \alpha_{j_{1}} S_{j_{1}, \ldots, j_{m}}^{i_{1}, \ldots, i_{n}}
$$

where $S_{j_{1}, \ldots, j_{m}}^{i_{1}, \ldots, i_{n}} \in \mathbf{X}(\mathcal{T})$ are diagonal operators, uniquely determined by

$$
\begin{aligned}
& S_{j_{1}, \ldots, j_{m}}^{i_{1}, \ldots, i_{n}} \chi_{t}=0, \quad t \notin \mathcal{T} \alpha_{j_{m}} \cdots \alpha_{j_{1}}, \\
& S_{j_{1}, \ldots, j_{m}}^{i_{1}, \ldots, i_{n}} \chi_{t \alpha_{j_{m}} \cdots \alpha_{j_{1}}}=\left[S \chi_{t \alpha_{j_{m}} \cdots \alpha_{j_{1}}}, \chi_{t \alpha_{i_{n}} \cdots \alpha_{i_{1}}}\right] \chi_{t \alpha_{j_{m}} \cdots \alpha_{j_{1}}}, \quad t \in \mathcal{T} .
\end{aligned}
$$

Proof. Let $f \in \ell_{2}(\mathcal{T})$ and $t \in \mathcal{T}$ be fixed. Using the operators, defined by (3.6), (3.7), one can rewrite (3.2) as follows:

$$
\begin{gathered}
S f(t)=\sum_{u \in \mathcal{T}}\left[S \chi_{u}, \chi_{t}\right] f(u) \\
=\sum_{n, m=0}^{\infty} \sum_{\substack{1 \leq j_{1}, \ldots, j_{m} \leq q \\
t \bar{\gamma}^{n} \alpha_{j_{m}} \neq t \bar{\gamma}^{n-1}}}\left[S \chi_{t \bar{\gamma}^{n} \alpha_{j_{m}} \cdots \alpha_{j_{1}}}, \chi_{t}\right] f\left(t \bar{\gamma}^{n} \alpha_{j_{m}} \cdots \alpha_{j_{1}}\right) \\
=\sum_{n, m=0}^{\infty} \sum_{\substack{i_{1}, \ldots, i_{n} \leq q \\
j_{1}, \ldots, j_{m} \\
j_{m} \neq i_{n}}} \bar{\alpha}_{i_{1}} \cdots \bar{\alpha}_{i_{n}} \alpha_{j_{m}} \cdots \alpha_{j_{1}} S_{j_{1}, \ldots, j_{m}}^{i_{1}, \ldots, i_{n}} f(t),
\end{gathered}
$$

and we obtain (3.5), where the convergence is point-wise.

Furthermore, let $t \in \mathcal{T}$ be fixed and let $S \in \mathbf{X}(\mathcal{T})$ be of the form 3.5, where the coefficients $S_{j_{1}, \ldots, j_{m}}^{i_{1}, \ldots, i_{n}}$ are diagonal, then

$$
\begin{aligned}
& S \chi_{t}=\sum_{n, m=0}^{\infty} \sum_{\substack{i_{1}, \ldots, i_{n} \\
j_{1}, \ldots, j_{m} \leq q \\
j_{m} \neq i_{n}}} \bar{\alpha}_{i_{1}} \cdots \bar{\alpha}_{i_{n}} \alpha_{j_{m}} \cdots \alpha_{j_{1}} S_{j_{1}, \ldots, j_{m}}^{i_{1}, \ldots, i_{n}} \chi_{t} \\
& =\sum_{n, m=0}^{\infty} \sum_{\substack{i_{1}, \ldots, i_{n} \leq q \\
j_{1}, \ldots, j_{m} \leq q \\
j_{m} \neq i_{n}}}\left[S_{j_{1}, \ldots, j_{m}}^{i_{1}, \ldots, i_{n}} \chi_{t}, \chi_{t}\right] \bar{\alpha}_{i_{1}} \cdots \bar{\alpha}_{i_{n}} \alpha_{j_{m}} \cdots \alpha_{j_{1}} \chi_{t} \\
& =\sum_{n, m=0}^{\infty} \sum_{\substack{1 \leq i_{1}, \ldots, i_{n} \leq q \\
i_{n} \neq \hat{i}_{m}}}\left[S_{\hat{i}_{1}, \ldots, \hat{i}_{m}}^{i_{1}, \ldots, i_{n}} \chi_{t}, \chi_{t}\right] \chi_{t \bar{\gamma}^{m} \alpha_{i_{n}} \cdots \alpha_{i_{1}}},
\end{aligned}
$$


where the indices $\hat{i}_{k}$ are determined by $t \bar{\gamma}^{k} \alpha_{\hat{i}_{k}}=t \bar{\gamma}^{k-1}$. Since the sum above is taken over $i_{n} \neq \hat{i}_{m}$, all the summands are mutually orthogonal and (3.7) follows. Thus, under the restriction (3.6), the coefficients $S_{j_{1}, \ldots, j_{m}}^{i_{1}, \ldots, i_{n}}$ are determined uniquely.

Proposition 3.3. The multiscale linear system (3.1) is causal if, and only if, the coefficients of the representation (3.5) for $S$ satisfy

$$
S_{j_{1}, \ldots, j_{m}}^{i_{1}, \ldots, i_{n}}=0 \text {, whenever } n<m .
$$

Proof. According to Definition 3.1] the multiscale linear system (3.1) is causal if and only if

$$
\left[S \chi_{u}, \chi_{t}\right]=0 \text {, whenever } t \npreceq u \text {. }
$$

Hence (3.8) follows immediately from (3.7).

Next we turn to the notion of stationarity. As in the classical case, this should mean translation-invariance. However, here the primitive downward shifts are oneto-one but not onto, while the primitive upward shift is onto, but not one-to-one. In particular, neither is a tree isometry (a tree isometry is a graph automorphism which preserves distances) and hence is not suitable for the role of a translation. Instead, we shall say that a tree isometry $\tau: \mathcal{T} \mapsto \mathcal{T}$ is a primitive translation if for every $t \in \mathcal{T}$

$$
t \tau \bar{\gamma} \asymp t .
$$

Let us analyze the structure of a primitive translation $\tau$. First of all, we note that, since $\tau$ is a tree isometry,

$$
\operatorname{dist}(t \bar{\gamma} \tau, t \tau)=1
$$

According to (3.9),

$$
t \bar{\gamma} \tau \bar{\gamma} \asymp t \bar{\gamma} \preceq t \asymp t \tau \bar{\gamma}
$$

and hence

$$
t \bar{\gamma} \tau \preceq t \tau .
$$

Therefore, by definition, $t \bar{\gamma} \tau=t \tau \bar{\gamma}$ and we conclude that $\tau$ commutes with the primitive upward shift:

$$
\tau \bar{\gamma}=\bar{\gamma} \tau .
$$

Furthermore, we observe that $\tau \bar{\gamma}$ must have a fixed point. Indeed, let $t \in \mathcal{T}$ and let

$$
v=t \wedge t \tau=t \bar{\gamma}^{n}=t \tau \bar{\gamma}^{n+1}, \text { where } n=\operatorname{dist}(t \wedge t \tau, t) .
$$

Then, by (3.10),

$$
v \tau \bar{\gamma}=t \bar{\gamma}^{n} \tau \bar{\gamma}=t \tau \bar{\gamma}^{n+1}=v
$$

Thus we obtain a unique sequence of nodes

$$
v_{j}=v \tau^{j}, \quad j \in \mathbb{Z},
$$

satisfying

$$
v_{j} \bar{\gamma}=v_{j-1}, v_{j} \tau=v_{j+1}
$$


It is called the skeleton of the primitive translation $\tau$. Each node $v_{j}$ of the skeleton corresponds to the non-homogeneous tree $\mathcal{T}_{v_{j}}^{+}$, which is the maximal connected subgraph of $\mathcal{T}$, satisfying

$$
\mathcal{T}_{v_{j}}^{+} \bigcap\left\{v_{j-1}, v_{j}, v_{j+1}\right\}=v_{j}
$$

It is mapped isometrically by $\tau$ onto $\mathcal{T}_{v_{j+1}}^{+}$.

The operator of convolution with a primitive translation is unitary on $\ell_{2}(\mathcal{T})$. By abuse of notation, we denote both the group of the tree isometries, generated by primitive translations, and the group of the corresponding convolution operators by $\mathbf{A}(\mathcal{T})$.

Definition 3.4. The multiscale linear system (3.1) is said to be stationary if $S$ commutes with every $\tau \in \mathbf{A}(\mathcal{T})$.

Lemma 3.5. The multiscale linear system (3.1) is stationary if and only if the value of the scalar product $\left[S \chi_{u}, \chi_{t}\right]$ depends only on $\operatorname{dist}(t \wedge u, t)$ and $\operatorname{dist}(t \wedge u, u)$.

Proof. First of all, we note that, according to Definition 3.4 the multiscale linear system (3.1) is stationary if and only if for every pair of nodes $t, u \in \mathcal{T}$ and every $\tau \in \mathbf{A}(\mathcal{T})$

$$
\left[S \chi_{u}, \chi_{t}\right]=\left[S \chi_{u \tau}, \chi_{t \tau}\right]
$$

So let us assume that $\left[S \chi_{u}, \chi_{t}\right]$ depends only on $n=\operatorname{dist}(t \wedge u, t)$ and $m=$ $\operatorname{dist}(t \wedge u, u)$ and let $\tau \in \mathbf{A}(\mathcal{T})$. Without loss of generality, we can also assume that $\tau$ is a primitive translation. Let us fix now a pair $t, u$, then it follows from (3.9) that

Since

$$
(t \wedge u) \tau=t \bar{\gamma}^{n}=u \bar{\gamma}^{m}
$$

$$
\operatorname{dist}(t \tau, u \tau)=\operatorname{dist}(t, u)=m+n,
$$

we conclude that

$$
(t \wedge u) \tau=(t \tau) \wedge(u \tau)
$$

and, therefore, (3.11) holds true.

Conversely, let us assume that the multiscale linear system (3.1) is stationary. Then, in view of (3.11), it suffices to prove that for any two pairs of nodes $t, u$ and $t^{\prime}, u^{\prime}$, satisfying

$$
\operatorname{dist}(t \wedge u, t)=\operatorname{dist}\left(t^{\prime} \wedge u^{\prime}, t^{\prime}\right)=n, \operatorname{dist}(t \wedge u, u)=\operatorname{dist}\left(t^{\prime} \wedge u^{\prime}, u^{\prime}\right)=m,
$$

there exists $\tau \in \mathbf{A}(\mathcal{T})$, such that

$$
t \tau=t^{\prime}, u \tau=u^{\prime}
$$

Such an isometry can be constructed as follows. Denote

$$
\operatorname{dist}\left((t \wedge u) \wedge\left(t^{\prime} \wedge u^{\prime}\right), t \wedge u\right)=k, \operatorname{dist}\left((t \wedge u) \wedge\left(t^{\prime} \wedge u^{\prime}\right), t^{\prime} \wedge u^{\prime}\right)=p .
$$

In the case $t=u$ we choose arbitrary primitive translations $\tau_{1}$ and $\tau_{2}$, such that

$$
t \tau_{1} \bar{\gamma}=t, t^{\prime} \tau_{2} \bar{\gamma}=t^{\prime},
$$

and set

$$
\tau=\tau_{1}^{-k} \tau_{2}^{p}
$$


In the case $t \neq u$ we assume, without loss of generality, that $n \neq 0$ and choose $\tau_{1}$ as above. Then

$$
t \tau_{1}^{-n-k}=t \bar{\gamma}^{-n-k}=(t \wedge u) \wedge\left(t^{\prime} \wedge u^{\prime}\right)=t^{\prime} \bar{\gamma}^{-n-p} .
$$

Now let $t^{\prime}$ belong to the skeleton of a primitive translation $\tau_{2}$. Then

$$
\begin{gathered}
u \tau_{1}^{-n-k} \in \mathcal{T}_{t^{\prime} \tau_{2}^{-2 n-p}}^{+}, \quad u^{\prime} \in \mathcal{T}_{t^{\prime} \tau_{2}^{-n}}^{+}, \\
\operatorname{dist}\left(u \tau_{1}^{-n-k}, t^{\prime} \tau_{2}^{-2 n-p}\right)=\operatorname{dist}\left(u^{\prime}, t^{\prime} \tau_{2}^{-n}\right)=m .
\end{gathered}
$$

Thus $\tau_{2}$ can be chosen so that

$$
u \tau_{1}^{-n-k} \tau_{2}^{n+p}=u^{\prime}
$$

and we set

$$
\tau=\tau_{1}^{-n-k} \tau_{2}^{n+p}
$$

Remark 3.6. In view of the formula (3.7), Lemma 3.5 implies that the multiscale linear system (3.1) is stationary if, and only if, each coefficient $S_{j_{1}, \ldots, j_{m}}^{i_{1}, \ldots, i_{n}}$ in the series (3.5) has a constant (except for normalizing zeroes - see (3.6)) diagonal and, moreover, for $t \in \mathcal{T} \alpha_{j_{1}} \cdots \alpha_{j_{m}}$ the diagonal entry $\left[S_{j_{1}, \ldots, j_{m}}^{i_{1}, \ldots, i_{n}} \chi_{t}, \chi_{t}\right]$ depends only on $n, m$. The first condition is the multiscale analogue of the Toeplitz condition. Unlike the discrete time case, here it is weaker than the stationarity condition.

Theorem 3.7. The multiscale linear system (3.1) is stationary if, and only if,

$$
S \in \overline{\operatorname{span}}_{\mathbb{C}}\left\{\bar{\gamma}^{n} \gamma^{m}: n, m \in \mathbb{Z}_{+}\right\},
$$

where the closure is taken in the point-wise sense. In this case, the system (3.1) is also causal if, and only if,

$$
S \in \overline{\operatorname{span}}_{\mathbb{C}}\left\{\bar{\gamma}^{n} \gamma^{m}: n \geq m\right\} .
$$

Proof. Let us assume first that the multiscale linear system (3.1) is stationary. Then, since

$$
\alpha_{j_{m}} \cdots \alpha_{j_{1}}=\alpha_{j_{m}} \cdots \alpha_{j_{1}} \pi_{j_{1}, \ldots, j_{m}},
$$

where $\pi_{j_{1}, \ldots, j_{m}}$ denotes the orthogonal projection onto the subspace of signals supported in $\mathcal{T} \alpha_{j_{m}} \cdots \alpha_{j_{1}}$, Remark [3.6 means that the representation (3.5) can be rewritten as

$$
S=\sum_{n, m \in \mathbb{Z}^{+}} \sum_{\substack{i_{1}, \ldots, i_{n} \\ j_{1}, \ldots, j_{m} \leq q \\ j_{m} \neq i_{n}}} \bar{\alpha}_{i_{1}} \cdots \bar{\alpha}_{i_{n}} \alpha_{j_{m}} \cdots \alpha_{j_{1}} s_{n, m}, \quad s_{n, m} \in \mathbb{C} .
$$

But the partial sums of this series belong to $\operatorname{span}_{\mathbb{C}}\left\{\bar{\gamma}^{n} \gamma^{m}: n, m \in \mathbb{Z}_{+}\right\}$, since (3.4) leads to

$$
\sum_{\substack{1 \leq i_{1}, \ldots, i_{n} \\ j_{1}, \ldots, j_{m} \leq q \\ j_{m} \neq i_{n}}} \bar{\alpha}_{i_{1}} \cdots \bar{\alpha}_{i_{n}} \alpha_{j_{m}} \cdots \alpha_{j_{1}}=q^{\frac{m+n}{2}} \bar{\gamma}^{n} \gamma^{m}-q^{\frac{m+n-2}{2}} \bar{\gamma}^{n-1} \gamma^{m-1} .
$$

If the system (3.1) is also causal then, according to Proposition 3.3 we have $s_{n, m}=$ 0 for $n<m$, hence

$$
S \in \overline{\operatorname{span}}_{\mathbb{C}}\left\{\bar{\gamma}^{n} \gamma^{m}: n \geq m\right\} .
$$


In order to prove the converse statements, we note first that if $S$ is of the form $S=\bar{\gamma}^{n} \gamma^{m}$ then, because of (3.10) and the fact that

$$
\tau \in \mathbf{A}(\mathcal{T}) \Longrightarrow \tau^{*}=\tau^{-1} \in \mathbf{A}(\mathcal{T}),
$$

the system (3.1) is stationary. In the case $n \geq m$ it is also causal, as follows from Proposition 3.3 It only remains to observe that, in view of our Definitions $3.1,3.4$ and the fact that each operator $\tau \in \mathbf{A}(\mathcal{T})$ is a convolution operator, the properties of causality and stationarity are preserved when passing to the point-wise limit.

\section{Stationary multiscale Systems And NON-STATIONARY DisCRETE Time SYSTEMS}

The main goal of the present work is to investigate the multiscale systems of the form (3.1) which are both causal and stationary. We denote the Banach algebra of corresponding operators $S$ by $\mathbf{U}(\mathcal{T})$. According to Theorem 3.7

$$
\mathbf{U}(\mathcal{T})=\overline{\operatorname{span}}_{\mathbb{C}}\left\{\bar{\gamma}^{n} \sigma_{m}: n, m \in \mathbb{Z}_{+}\right\},
$$

where the closure is taken in the point-wise sense and

$$
\sigma_{m}=\bar{\gamma}^{m} \gamma^{m}, \quad m \in \mathbb{Z}_{+} .
$$

We note that the self-adjoint operator $\sigma_{m} \in \mathbf{U}(\mathcal{T})$, defined by (4.2), is just the average

$$
\sigma_{m} f(t)=\frac{1}{q^{m}} \sum_{\substack{u \asymp t \\ \operatorname{dist}(u, t) \leq 2 m}} f(u)
$$

and hence acts on the subspace of functions, supported in a horocycle. Furthermore, since $\bar{\gamma}$ is an isometry,

$$
\sigma_{m} \sigma_{n}=\sigma_{m \vee n},
$$

where $m \vee n$ denotes the maximal of two integers $m$ and $n$. Hence it is more convenient to consider

$$
\omega_{m}=\sigma_{m}-\sigma_{m+1}, \quad m \in \mathbb{Z}_{+} .
$$

Theorem 4.1. The space $\ell_{2}(\mathcal{T})$ admits the orthogonal decomposition

$$
\ell_{2}(\mathcal{T})=\bigoplus_{m=0}^{\infty} \mathcal{W}_{m}
$$

where the orthogonal projections $\omega_{m}: \ell_{2}(\mathcal{T}) \mapsto \mathcal{W}_{m}$ are given by (4.5). Each subspace $\mathcal{W}_{m}$ is mapped isometrically onto $\mathcal{W}_{m+1}$ by the upward shift operator $\bar{\gamma}$.

Proof. It follows immediately from (4.2) (4.4) and (4.5) that

$$
\omega_{m}^{*}=\omega_{m}, \omega_{m} \omega_{n}=\delta_{m, n} \omega_{n} .
$$

In order to show that $\sum_{m=0}^{\infty} \omega_{m}$ converges in the strong operator topology to the identity operator $I$, it suffices to note that

$$
\sum_{m=0}^{n} \omega_{m}=I-\sigma_{n+1}
$$


and that, in view of (4.3), the sequence $\sigma_{n}$ converges in the strong operator topology to 0.

Finally, we have to show that

$$
\bar{\gamma} \mathcal{W}_{m}=\mathcal{W}_{m+1}, \quad m \in \mathbb{Z}_{+} .
$$

But it follows from (3.4), (4.2) and (4.5) that

$$
\omega_{m+1}=\bar{\gamma} \omega_{m} \gamma \text { and } \bar{\gamma} \omega_{m}=\omega_{m+1} \bar{\gamma} .
$$

It follows from Theorem 4.1 that an operator $S \in \mathbf{X}(\mathcal{T})$ can be viewed as an operator matrix with blocks corresponding to the orthogonal decomposition (4.6) and thus the corresponding multiscale linear system (3.1) can be treated as a (nonstationary, in general) discrete time system (see [2] and Section 21). Let us investigate the causal stationary multiscale linear systems from this point of view.

Proposition 4.2. Let $S \in \mathbf{X}(\mathcal{T})$. Then $S \in \mathbf{U}(\mathcal{T})$ if, and only if,

$$
\omega_{m} S \omega_{n}= \begin{cases}\bar{\gamma}^{m-n} \omega_{n} s_{m, n}, & m \geq n, \\ 0, & \text { otherwise, }\end{cases}
$$

where $s_{m, n} \in \mathbb{C}$.

Proof. Let us assume that $S \in \mathbf{U}(\mathcal{T})$. Then, by (4.1), there exists a sequence $S_{k} \in \operatorname{span}_{\mathbb{C}}\left\{\bar{\gamma}^{j} \sigma_{i}: i, j \in \mathbb{Z}_{+}\right\}$which converges to $S$ point-wise. (4.7) implies that

$$
\omega_{m} S_{k} \omega_{n}= \begin{cases}\bar{\gamma}^{m-n} \omega_{n} s_{m, n, k}, & m \geq n, \\ 0, & \text { otherwise, }\end{cases}
$$

where $s_{m, n, k} \in \mathbb{C}$. Since for every $m \in \mathbb{Z}_{+}$and $t \in \mathcal{T} \omega_{m} \chi_{t}$ has a finite support,

$$
\lim _{k \rightarrow \infty} \omega_{m} S_{k} \omega_{n}=\omega_{m} S \omega_{n} \text { point-wise. }
$$

In particular, there exists $\lim _{k \rightarrow \infty} s_{m, n, k}$, which we can denote by $s_{m, n}$ and thus obtain (4.8).

Conversely, if (4.8) holds, then by Theorem 4.1 we can represent $S$ as the strongly converging series

$$
S=\sum_{m=0}^{\infty} \omega_{m} S=\sum_{m=0}^{\infty} \sum_{n=0}^{m} \omega_{m} S \omega_{n}
$$

Since the convergence in the strong operator topology implies the point-wise convergence, it suffices to apply Theorem [3.7 to complete the proof.

From Proposition 4.2 it follows that a multiscale system (3.1) is stationary and causal if, and only if, $S$ is "triangular" with respect to the orthogonal decomposition (4.6) - that is, for every $n \in \mathbb{Z}_{+}$the subspace of piece-wise constant functions $\oplus_{m \geq n} \mathcal{W}_{m}=\operatorname{ran} \sigma_{n}$ is $S$-invariant ${ }^{1}-$ and, moreover, the blocks of $S$ are complex constants. However, the subspaces $\mathcal{W}_{m}$ are infinite-dimensional and hence the only Hilbert-Schmidt element of $\mathbf{U}(\mathcal{T})$ is 0 . Nevertheless, we can adapt the techniques,

\footnotetext{
${ }^{1}$ In the language of nest algebras (see 5 19]) $S$ is in the nest algebra associated with the nest $\left\{\oplus_{m \geq n} \mathcal{W}_{m}: n \in \mathbb{Z}_{+}\right\}$.
} 
developed for the non-stationary discrete time systems, to the present setting.

In order to formulate the appropriate analogue of Theorem 2.1] we consider the space of operators

$$
\mathbb{K}=\left\{\mathbf{c}=\sum_{m=0}^{\infty} c_{m} \omega_{m}: c_{m} \in \mathbb{C}, \sup _{m \in \mathbb{Z}_{+}}\left|c_{m}\right|<\infty,\right\}
$$

where the convergence is in the strong operator topology. According to Theorem 4.1 and Proposition $4.2 \mathbb{K}$ is a subalgebra of $\mathbf{U}(\mathcal{T})$ and, moreover, a commutative $\mathbb{C}^{*}$-algebra ${ }^{2}$, isometric to $\ell_{\infty}\left(\mathbb{Z}^{+}\right)$. For $\mathbf{c} \in \mathbb{K}$ we shall use the notation

$$
\overline{\mathbf{c}}=\mathbf{c}^{*}=\sum_{m=0}^{\infty} \bar{c}_{m} \omega_{m} .
$$

We also introduce the notion of the row-wise (with respect to the orthogonal decomposition (4.6)) convergence: we shall say that a sequence of $S_{n} \in \mathbf{X}(\mathcal{T})$ converges row-wise to $S \in \mathbf{X}(\mathcal{T})$ if for every $m \in \mathbb{Z}_{+}$the sequence $\omega_{m} S_{n}$ converges to $\omega_{m} S$ in the operator norm.

Theorem 4.3. Let $S \in \mathbf{X}(\mathcal{T})$. Then $S \in \mathbf{U}(\mathcal{T})$ if, and only if, it can be represented as a row-wise converging series

$$
S=\sum_{k=0}^{\infty} \bar{\gamma}^{k} \mathbf{s}_{k}, \quad \mathbf{s}_{k} \in \mathbb{K} .
$$

In this case the operators $\mathbf{s}_{k}$ are determined uniquely by

$$
\omega_{n} \mathbf{s}_{k}=\gamma^{k} \omega_{n+k} S \omega_{n}, \quad n, k \in \mathbb{Z}_{+},
$$

and it holds that

$$
\sum_{k=0}^{\infty} \overline{\mathbf{s}}_{k} \mathbf{s}_{k} \omega_{n}=\frac{q^{n+1}}{q-1}\left\|S \omega_{n} \chi_{t}\right\|^{2} \omega_{n}, \quad t \in \mathcal{T}, n \in \mathbb{Z}^{+} .
$$

Proof. First, let us assume that there exists a sequence $\mathbf{s}_{k} \in \mathbb{K}$ such that the series $\sum_{k} \bar{\gamma}^{k} \mathbf{s}_{k}$ converges row-wise to an operator $S \in \mathbf{X}(\mathcal{T})$. Then for any $m, n \in \mathbb{Z}_{+}$

$$
\sum_{k=0}^{\infty} \omega_{m} \bar{\gamma}^{k} \mathbf{s}_{k} \omega_{n}=\omega_{m} S \omega_{n}
$$

Then, since

$$
\omega_{m} \bar{\gamma}^{k} \mathbf{s}_{k} \omega_{n}=\delta_{k, m-n} \bar{\gamma}^{k} \mathbf{s}_{k} \omega_{n},
$$

$S \in \mathbf{U}(\mathcal{T})$ by Proposition 4.2. Moreover,

$$
\bar{\gamma}^{k} \mathbf{s}_{k} \omega_{n}=\omega_{n+k} S \omega_{n}, \quad n, k \in \mathbb{Z}_{+},
$$

hence (4.10) holds true.

Conversely, let us assume that $S \in \mathbf{U}(\mathcal{T})$ and let $m \in \mathbb{Z}_{+}$. Then, according to Proposition 4.2 we can define $\mathbf{s}_{k} \in \mathbb{K}$ by (4.10) and observe that

$$
\omega_{m} S=\sum_{n=0}^{m} \omega_{m} S \omega_{n}=\sum_{n=0}^{m} \omega_{m} \bar{\gamma}^{m-n} \mathbf{s}_{m-n}=\sum_{n=0}^{m} \omega_{m} \bar{\gamma}^{n} \mathbf{s}_{n}=\sum_{n=0}^{\infty} \omega_{m} \bar{\gamma}^{n} \mathbf{s}_{n} .
$$

\footnotetext{
${ }^{2}$ For background on $\mathbb{C}^{*}$-algebras we refer the reader to 18 .
} 
Finally,

$$
\begin{aligned}
& \left\|S \omega_{n} \chi_{t}\right\|^{2} \omega_{n}=\sum_{m=n}^{\infty}\left\|\omega_{m} S \omega_{n} \chi_{t}\right\|^{2} \omega_{n}=\sum_{m=n}^{\infty}\left\|\bar{\gamma}^{m-n} \mathbf{s}_{m-n} \omega_{n} \chi_{t}\right\|^{2} \omega_{n} \\
& =\sum_{m=0}^{\infty}\left\|\mathbf{s}_{m} \omega_{n} \chi_{t}\right\|^{2} \omega_{n}=\left\|\omega_{n} \chi_{t}\right\|^{2} \sum_{k=0}^{\infty} \overline{\mathbf{s}}_{k} \mathbf{s}_{k} \omega_{n}=\left(\frac{1}{q^{n}}-\frac{1}{q^{n+1}}\right) \sum_{k=0}^{\infty} \overline{\mathbf{s}}_{k} \mathbf{s}_{k} \omega_{n},
\end{aligned}
$$

and we obtain (4.11).

Following the analogy with the non-stationary setting, we consider the following ideal of $\mathbb{K}$ :

$$
\mathbb{K}_{2}=\left\{\mathbf{c} \in \mathbb{K}: \sum_{m=0}^{\infty}\left|c_{m}\right|^{2}<\infty\right\}
$$

It is a Hilbert space, isometric to $\ell_{2}\left(\mathbb{Z}^{+}\right)$. We also consider the $\mathbb{K}$-module ${ }^{3}$ :

$$
\mathbf{H}_{2}(\mathcal{T})=\left\{S=\sum_{k=0}^{\infty} \bar{\gamma}^{k} \mathbf{s}_{k}: \mathbf{s}_{k} \in \mathbb{K}_{2}, \sum_{k=0}^{\infty}\left\|\mathbf{s}_{k}\right\|_{2}^{2}<\infty\right\} .
$$

At this point we consider the power series in the definition above as formal. However, we shall see later on (Proposition 4.5) that such a series converges in the operator norm. This is the analogue of the space of Hilbert-Schmidt operators in the present setting.

Proposition 4.4. The $\mathbb{K}$-module $\mathbf{H}_{2}(\mathcal{T})$, considered as a vector space over $\mathbb{C}$ with the scalar product

$$
[F, G]=\sum_{k=0}^{\infty}\left[\mathbf{f}_{k}, \mathbf{g}_{k}\right]
$$

is a Hilbert space.

Proof. The proof is the same as in the case of Hilbert-Schmidt operators, hence we shall give only an outline. Since the Cauchy-Schwarz inequality holds in $\mathbb{K}_{2}$, the inner product (4.12) is well-defined in the whole of $\mathbf{H}_{2}(\mathcal{T})$, which is, therefore, a pre-Hilbert space. Hence, the Cauchy-Schwarz inequality holds in $\mathbf{H}_{2}(\mathcal{T})$ as well, and

$$
\|F\|_{2}=\sqrt{[F, F]}
$$

is a well-defined norm. The completeness of $\mathbf{H}_{2}(\mathcal{T})$ with respect to this norm can now be proved, using the triangle inequality.

Proposition 4.5. The following hold:

(1) The Hilbert space $\mathbf{H}_{2}(\mathcal{T})$ is contractively contained in $\mathbf{U}(\mathcal{T})$.

(2) Let $S \in \mathbf{U}(\mathcal{T})$ and $t \in \mathcal{T}$. Then $S \in \mathbf{H}_{2}(\mathcal{T})$ if, and only if,

$$
\sum_{k=0}^{\infty} q^{k}\left\|S \omega_{k} \chi_{t}\right\|^{2}<\infty
$$

In this case the expression above is equal to $\left(1-\frac{1}{q}\right)\|S\|_{2}^{2}$.

\footnotetext{
${ }^{3}$ For background on modules over a $\mathbb{C}^{*}$-algebra see [20], [21], [23].
} 
Proof.

(1) In view of completeness of $\mathbf{H}_{2}(\mathcal{T}), \mathbf{U}(\mathcal{T})$ and $\ell_{2}(\mathcal{T})$, it suffices to consider $S \in \mathbf{H}_{2}(\mathcal{T})$ such that the coefficients $\mathbf{s}_{k} \in \mathbb{K}_{2}$ are different from zero only for a finite number of indices $k$. Then $S \in \mathbf{U}(\mathcal{T})$ and we have

$$
\begin{aligned}
&\|S f\|^{2}=\sum_{n=0}^{\infty}\left\|\omega_{n} S f\right\|^{2} \leq \sum_{n=0}^{\infty}\left(\sum_{k=0}^{n}\left\|\mathbf{s}_{k} \omega_{n-k} f\right\|\right)^{2} \\
& \leq \sum_{n=0}^{\infty}\left(\sum_{k=0}^{n}\left\|\mathbf{s}_{k} \omega_{n-k}\right\|^{2}\right)\left(\sum_{m=0}^{n}\left\|\omega_{n-m} f\right\|^{2}\right) \\
& \leq \sum_{m=0}^{\infty}\left\|\omega_{m} f\right\|^{2} \sum_{n, k=0}^{\infty}\left\|\mathbf{s}_{k} \omega_{n}\right\|^{2}=\|f\|^{2}\|S\|_{2}^{2} .
\end{aligned}
$$

(2) It follows from (4.11) that

$$
\|S\|_{2}^{2}=\sum_{k=0}^{\infty}\left\|\mathbf{s}_{k}\right\|_{2}^{2}=\frac{q}{q-1} \sum_{k=0}^{\infty} q^{k}\left\|S \omega_{k} \chi_{t}\right\|^{2},
$$

whenever either right-hand side or left-hand side is finite.

Remark 4.6. A consequence of Proposition 4.5 is that $\mathbf{H}_{2}(\mathcal{T})$ is a left ideal in $\mathbf{U}(\mathcal{T})$ and, moreover, for any $S \in \mathbf{U}(\mathcal{T})$ and $F \in \mathbf{H}_{2}(\mathcal{T})$ the inequality

$$
\|S F\|_{2} \leq\|S\|\|F\|_{2}
$$

holds true.

\section{Point evaluation}

In this section we exploit the analogy with the non-stationary setting to associate the elements of $\mathbf{U}(\mathcal{T})$ with maps from $\mathbb{K}$ into itself.

Recalling the identity (4.7) and observing that $\omega_{0} \bar{\gamma}=0$, we conclude that for every $\mathbf{c} \in \mathbb{K}$ there exists an element

$$
\mathbf{c}^{(1)}=\sum_{n=0}^{\infty} \omega_{n} c_{n+1} \in \mathbb{K}
$$

such that

$$
\mathbf{c} \bar{\gamma}=\bar{\gamma} \mathbf{c}^{(1)}
$$

We note that $\left.\| \mathbf{c}^{(1)}\right)\|\leq\| \mathbf{c} \|$ and $(\mathbf{c d})^{(1)}=\mathbf{c}^{(1)} \mathbf{d}^{(1)}$. Furthermore, we introduce the following notation:

$$
\begin{gathered}
\mathbf{c}^{(0)}=\mathbf{c}, \quad \mathbf{c}^{(n+1)}=\left(\mathbf{c}^{(n)}\right)^{(1)}, \\
\mathbf{c}^{[0]}=1, \quad \mathbf{c}^{[n+1]}=\mathbf{c}^{[n]} \mathbf{c}^{(n)}, \\
\rho(\mathbf{c})=\limsup _{n \rightarrow \infty}\left\|\mathbf{c}^{[n]}\right\|^{\frac{1}{n}}, \\
\mathbb{D}(\mathcal{T})=\{\mathbf{c} \in \mathbb{K}: \rho(\mathbf{c})<1\} .
\end{gathered}
$$

The set $\mathbb{D}(\mathcal{T})$ is the counterpart of the open unit disk in the present setting. 
Definition 5.1. Let $S \in \mathbf{U}(\mathcal{T})$ be given. For $\mathbf{c} \in \mathbb{D}(\mathcal{T})$ we define the point evaluation of $S$ at $\mathbf{c}$ by

$$
S(\mathbf{c})=\sum_{n=0}^{\infty} \mathbf{c}^{[n]} \mathbf{s}_{n} .
$$

We note that (5.1) is the analogue of (2.2). We claim that the point evaluation (5.1) is well-defined. Indeed, the convergence of the series (5.1) in $\mathbb{K}$ follows from Theorem 4.3 Also, if $S \in \mathbf{U}(\mathcal{T})$ is such that for every $\mathbf{c} \in \mathbb{D}(\mathcal{T}) S(\mathbf{c})=0$, then, in particular,

$$
S(0)=S\left(\omega_{k}\right)=S\left(\omega_{k}+\omega_{k+1}\right)=\ldots=0 .
$$

Hence

$$
\mathbf{s}_{0}=0, \mathbf{s}_{1} \omega_{k}=0, \mathbf{s}_{2} \omega_{k}=0, \ldots
$$

and $\mathbf{s}_{n}=0$ for $n=0,1,2, \ldots$ Furthermore, if $F \in \mathbf{H}_{2}(\mathcal{T})$ then for every $\mathbf{c} \in \mathbb{D}(\mathcal{T})$ $F(\mathbf{c}) \in \mathbb{K}_{2}$. We list several other properties of the point evaluation in the following

Lemma 5.2. The following hold:

(1) Let $F, G \in \mathbf{U}(\mathcal{T})$, $\mathbf{p}, \mathbf{q} \in \mathbb{K}, \mathbf{c} \in \mathbb{D}(\mathcal{T})$ and assume that $\mathbf{k} \in \mathbb{K}$ is invertible. Then

$$
\begin{aligned}
(F \mathbf{p}+G \mathbf{q})(\mathbf{c}) & =F(\mathbf{c}) \mathbf{p}+G(\mathbf{c}) \mathbf{q} \\
(F G)(\mathbf{c}) & =(F(\mathbf{c}) G)(\mathbf{c}) \\
\left(\bar{\gamma}^{n} F\right)(\mathbf{c}) & =\mathbf{c}^{[n]} F\left(\mathbf{c}^{(n)}\right) \\
(\mathbf{k} F)(\mathbf{c}) & =F\left(\mathbf{k}^{(1)} \mathbf{k}^{-1} \mathbf{c}\right) \mathbf{k} .
\end{aligned}
$$

(2) Let $F \in \mathbf{H}_{2}(\mathcal{T}), \mathbf{c} \in \mathbb{D}(\mathcal{T})$. Then

$$
F-F(\mathbf{c})=(\bar{\gamma}-\mathbf{c}) G,
$$

where $G \in \mathbf{H}_{2}(\mathcal{T})$ is given by

$$
G=\sum_{n, k=0}^{\infty} \bar{\gamma}^{n}\left(\mathbf{c}^{(n+1)}\right)^{[k]} \mathbf{f}_{n+k+1} .
$$

Proof.

(1) The relation (5.2) follows immediately from Definition 5.1 Furthermore,

$$
\begin{aligned}
\left(\bar{\gamma}^{n} F\right)(\mathbf{c})=\left(\sum_{k=0}^{\infty} \bar{\gamma}^{n+k} \mathbf{f}_{k}\right)(\mathbf{c})=\sum_{k=0}^{\infty} \mathbf{c}^{[n+k]} \mathbf{f}_{k} \\
=\sum_{k=0}^{\infty} \mathbf{c}^{[k]}\left(\mathbf{c}^{[n]}\right)^{(k)} \mathbf{f}_{k}=\left(\sum_{k=0}^{\infty} \bar{\gamma}^{k}\left(\mathbf{c}^{[n]}\right)^{(k)} \mathbf{f}_{k}\right)(\mathbf{c}) \\
=\left(\mathbf{c}^{[n]} \sum_{k=0}^{\infty} \bar{\gamma}^{k} \mathbf{f}_{k}\right)(\mathbf{c})=\left(\bar{\gamma}^{n}(\mathbf{c}) F\right)(\mathbf{c}),
\end{aligned}
$$

and, in view of (5.2), we obtain (5.3). Analogously,

$$
\left(\bar{\gamma}^{n} F\right)(\mathbf{c})=\sum_{k=0}^{\infty} \mathbf{c}^{[n+k]} \mathbf{f}_{k}=\sum_{k=0}^{\infty}\left(\mathbf{c}^{(n)}\right)^{[k]} \mathbf{f}_{k} \mathbf{c}^{[n]},
$$


and we obtain (5.4). Finally, we note that

$$
\left(\mathbf{k} \bar{\gamma}^{n}\right)(\mathbf{c})=\mathbf{c}^{[n]} \mathbf{k}^{(n)}=\left(\mathbf{c k}^{(1)} \mathbf{k}^{-1}\right)^{[n]} \mathbf{k}
$$

and (5.5) follows from (5.2).

(2) First, we have to check that the series (5.7) belongs to $\mathbf{H}_{2}(\mathcal{T})$. But

$$
\begin{gathered}
\sum_{n=0}^{\infty}\left\|\sum_{k=0}^{\infty}\left(\mathbf{c}^{(n+1)}\right)^{[k]} \mathbf{f}_{n+k+1}\right\|_{2}^{2} \leq \sum_{n=0}^{\infty}\left(\sum_{k=0}^{\infty}\left\|\mathbf{c}^{[k]}\right\|\left\|\mathbf{f}_{n+k+1}\right\|_{2}\right)^{2} \\
\leq \sum_{n=0}^{\infty}\left(\sum_{k=0}^{\infty}\left\|\mathbf{c}^{[k]}\right\|\right)\left(\sum_{m=0}^{\infty}\left\|\mathbf{c}^{[m]}\right\|\left\|\mathbf{f}_{n+m+1}\right\|_{2}^{2}\right) \\
\leq\left(\sum_{k=0}^{\infty}\left\|\mathbf{c}^{[k]}\right\|\right)^{2}\|F\|_{2}^{2}<\infty,
\end{gathered}
$$

since $\rho(\mathbf{c})<1$. Hence, indeed, $G \in \mathbf{H}_{2}(\mathcal{T})$.

Now we shall prove (5.6). Without loss of generality, we asssume that $F=\bar{\gamma}^{m} \mathbf{f}$, where $\mathbf{f} \in \mathbb{K}_{2}$. Then (5.7) means

$$
G=\sum_{n=1}^{m} \bar{\gamma}^{n-1}\left(\mathbf{c}^{(n)}\right)^{[m-n]} \mathbf{f} .
$$

In particular, for $m=1$ we have $G=\mathbf{f}$ and (5.6) holds. For $m \geq 2$ we have

$$
\left(\bar{\gamma}^{m}-\mathbf{c}^{[m]}\right) \mathbf{f}=(\bar{\gamma}-\mathbf{c}) \bar{\gamma}^{m-1} \mathbf{f}+\left(\bar{\gamma}^{m-1}-\mathbf{c}^{[m-1]}\right) \mathbf{f} \mathbf{c}^{(m-1)} .
$$

Applying induction on $m$, we obtain

$$
\begin{aligned}
\left(\bar{\gamma}^{m}-\mathbf{c}^{[m]}\right) \mathbf{f} & =(\bar{\gamma}-\mathbf{c})\left(\bar{\gamma}^{m-1}+\sum_{n=1}^{m-1} \bar{\gamma}^{n-1}\left(\mathbf{c}^{(n)}\right)^{[m-n-1]} \mathbf{c}^{(m-1)}\right) \mathbf{f} \\
& =(\bar{\gamma}-\mathbf{c})\left(\bar{\gamma}^{m-1}+\sum_{n=1}^{m-1} \bar{\gamma}^{n-1}\left(\mathbf{c}^{(n)}\right)^{[m-n]}\right) \mathbf{f}=(\bar{\gamma}-\mathbf{c}) G .
\end{aligned}
$$

A consequence of Lemma 5.2 is that $F \in \mathbf{H}_{2}(\mathcal{T})$ satisfies $F(\mathbf{c})=0$ if and only if $F$ is of the form (5.6), where $G \in \mathbf{H}_{2}(\mathcal{T})$.

Finally, we present an analogue of Cauchy's formula (and of formula (2.3) for the space $\mathbf{H}_{2}(\mathcal{T})$.

Theorem 5.3. Let $F \in \mathbf{H}_{2}(\mathcal{T})$, $\mathbf{c} \in \mathbb{D}(\mathcal{T})$. Then for every $\mathbf{k} \in \mathbb{K}_{2}$ it holds that

$$
[F(\mathbf{c}), \mathbf{k}]=\left[F, K_{\mathbf{c}} \mathbf{k}\right],
$$

where $K_{\mathbf{c}} \in \mathbf{U}(\mathcal{T})$ is given by

$$
K_{\mathbf{c}}=\sum_{n=0}^{\infty} \bar{\gamma}^{n} \overline{\mathbf{c}}^{[n]}=(1-\bar{\gamma} \overline{\mathbf{c}})^{-1} .
$$


Proof. Since $\mathbf{c} \in \mathbb{D}(\mathcal{T})$, there exists $\epsilon \in(0,1)$, such that for $n$ sufficiently large $\|\mathbf{c}\| \leq \epsilon^{n}$. It follows that the series (5.9) converges absolutely in $\mathbf{U}(\mathcal{T})$ and defines an operator $K_{\mathbf{c}}$, which satisfies

$$
K_{\mathbf{c}}(1-\bar{\gamma} \overline{\mathbf{c}})=(1-\bar{\gamma} \overline{\mathbf{c}}) K_{\mathbf{c}}=1 .
$$

The formula (5.8) follows immediately from (5.1).

\section{SCHUR MULTiPLIERS}

Let us recall that, in view of Proposition 4.5 and the subsequent remarks, for any $S \in \mathbf{U}(\mathcal{T})$ the multiplication operator $\mathcal{M}_{S} F=S F$ is a bounded linear operator from $\mathbf{H}_{2}(\mathcal{T})$ into itself.

Definition 6.1. $S \in \mathbf{U}(\mathcal{T})$ is called a Schur multiplier if the multiplication operator $\mathcal{M}_{S}$ is a contraction in $\mathbf{H}_{2}(\mathcal{T})$.

Theorem 6.2. An element $S \in \mathbf{U}(\mathcal{T})$ is a Schur multiplier if and only if the map $\mathcal{K}_{S}: \mathbb{D}(\mathcal{T}) \times \mathbb{D}(\mathcal{T}) \mapsto \mathbb{K}$, defined by

$$
\mathcal{K}_{S}(\mathbf{c}, \mathbf{d})=\sum_{n=0}^{\infty} \mathbf{c}^{[n]}(1-S(\mathbf{c}) \overline{S(\mathbf{d})})^{(n)} \overline{\mathbf{d}}^{[n]}
$$

is positive: for any $m \in \mathbb{Z}^{+}, \mathbf{c}_{0}, \ldots, \mathbf{c}_{m} \in \mathbb{D}(\mathcal{T}), \mathbf{k}_{0}, \ldots, \mathbf{k}_{m} \in \mathbb{K}_{2}$ it holds that

$$
\sum_{i, j=0}^{m}\left[\mathcal{K}_{S}\left(\mathbf{c}_{\mathbf{i}}, \mathbf{c}_{\mathbf{j}}\right) \mathbf{k}_{j}, \mathbf{k}_{i}\right] \geq 0 .
$$

Proof. Let $S \in \mathbf{U}(\mathcal{T}), \mathbf{c} \in \mathbb{D}(\mathcal{T}), \mathbf{k} \in \mathbb{K}_{2}$. Then

$$
\begin{array}{r}
\mathcal{M}_{S}^{*}\left(K_{\mathbf{c}} \mathbf{k}\right)=\sum_{n, m=0}^{\infty} \bar{\gamma}^{n} \omega_{m}\left[\mathcal{M}_{S}^{*}\left(K_{\mathbf{c}} \mathbf{k}\right), \bar{\gamma}^{n} \omega_{m}\right]=\sum_{n, m=0}^{\infty} \bar{\gamma}^{n}\left[K_{\mathbf{c}} \mathbf{k}, S \bar{\gamma}^{n} \omega_{m}\right] \omega_{m} \\
=\sum_{n, m=0}^{\infty} \bar{\gamma}^{n}\left[\mathbf{k},\left(S \bar{\gamma}^{n} \omega_{m}\right)(\mathbf{c})\right] \omega_{m}=\sum_{n, m=0}^{\infty} \bar{\gamma}^{n}\left[\overline{S(\mathbf{c})^{(n)}} \overline{\mathbf{c}}^{[n]} \mathbf{k}, \omega_{m}\right] \omega_{m} \\
=\sum_{n=0}^{\infty} \bar{\gamma}^{n} \overline{S(\mathbf{c})}^{(n)} \overline{\mathbf{c}}^{[n]} \mathbf{k} .
\end{array}
$$

It follows that

$$
\begin{aligned}
{\left[K_{\mathbf{c}_{2}} \mathbf{k}_{2}, K_{\mathbf{c}_{1}} \mathbf{k}_{1}\right]-\left[\mathcal{M}_{S}^{*}\left(K_{\mathbf{c}_{2}} \mathbf{k}_{2}\right), \mathcal{M}_{S}^{*}\left(K_{\mathbf{c}_{1}} \mathbf{k}_{1}\right)\right] } \\
\quad=\left[K_{\mathbf{c}_{2}}\left(\mathbf{c}_{1}\right) \mathbf{k}_{2}, \mathbf{k}_{1}\right]-\sum_{n=0}^{\infty}\left[\overline{S\left(\mathbf{c}_{2}\right)}{ }^{(n)} \overline{\mathbf{c}}_{2}{ }^{[n]} \mathbf{k}_{2}, \overline{S\left(\mathbf{c}_{1}\right)}{ }^{(n)} \overline{\mathbf{c}}_{1}{ }^{[n]} \mathbf{k}_{1}\right] \\
\quad=\sum_{n=0}^{\infty}\left[\mathbf{c}_{1}{ }^{[n]}\left(1-S\left(\mathbf{c}_{1}\right)^{(n)} \overline{S\left(\mathbf{c}_{2}\right)}{ }^{(n)}\right) \overline{\mathbf{c}}_{2}{ }^{[n]} \mathbf{k}_{2}, \mathbf{k}_{1}\right]=\left[\mathcal{K}_{S}\left(\mathbf{c}_{1}, \mathbf{c}_{2}\right) \mathbf{k}_{2}, \mathbf{k}_{1}\right],
\end{aligned}
$$

where $\mathcal{K}_{S}$ is given by (6.1). Since elements of the form

$$
F=\sum_{\ell=0}^{m} K_{\mathbf{c}_{\ell}} \mathbf{k}_{\ell}
$$


are dense in $\mathbf{H}_{2}(\mathcal{T}), S$ is a Schur multiplier if and only if for any such element $F$ it holds that

$$
[F, F]-\left[\mathcal{M}_{S}^{*} F, \mathcal{M}_{S}^{*} F\right] \geq 0
$$

which, in view of the computations above, is equivalent to the positivity condition (6.2).

Below, following the analysis of [2, p. 86-90], we give an example of a Schur multiplier. Let $\mathbf{a} \in \mathbb{D}(\mathcal{T})$. Then $K_{\mathbf{a}}(\mathbf{a}) \geq 0$ (in the sense of $\mathbb{K}$ ) and, since

$$
K_{\mathbf{a}}(\mathbf{a})=1+\overline{\mathbf{a}} \mathbf{a} K_{\mathbf{a}}(\mathbf{a})^{(1)},
$$

it is also invertible. Hence the element

$$
L_{\mathbf{a}}=K_{\mathbf{a}}(\mathbf{a})^{(1)} K_{\mathbf{a}}(\mathbf{a})^{-1}
$$

is also positive and invertible. Since

$$
L_{\mathbf{a}}^{[k]}=K_{\mathbf{a}}(\mathbf{a})^{(k)} K_{\mathbf{a}}(\mathbf{a})^{-1},
$$

we have $\rho\left(L_{\mathbf{a}}\right) \leq 1$.

Definition 6.3. The operator

$$
B_{\mathbf{a}}=(\bar{\gamma}-\mathbf{a})\left(1-L_{\mathbf{a}} \overline{\mathbf{a}} \bar{\gamma}\right)^{-1} \sqrt{L_{\mathbf{a}}} \in \mathbf{U}(\mathcal{T})
$$

is called the Blaschke factor, corresponding to $\mathbf{a}$.

Proposition 6.4. The multiplication operator $\mathcal{M}_{B_{\mathbf{a}}}$ is an isometry in $\mathbf{H}_{2}(\mathcal{T})$.

Proof. First of all, we note that, according to 4.12), $\mathcal{M}_{\bar{\gamma}}$ is an isometry in $\mathbf{H}_{2}(\mathcal{T})$, and that

$$
L_{\mathbf{a}}^{(m)}=L_{\mathbf{a}^{(m)}}, B_{\mathbf{a}} \bar{\gamma}^{m}=\bar{\gamma}^{m} B_{\mathbf{a}^{(m)}} .
$$

Hence it is enough to show that for any $m \in \mathbb{Z}^{+}$and $\mathbf{p}, \mathbf{q} \in \mathbb{K}_{2}$

$$
\left[B_{\mathbf{a}} \bar{\gamma}^{m} \mathbf{p}, B_{\mathbf{a}} \mathbf{q}\right]=\delta_{m, 0}[\mathbf{p}, \mathbf{q}] .
$$

To check this, we rewrite $B_{\mathbf{a}}$ in the form

$$
B_{\mathbf{a}}=\left(\bar{\gamma} K_{\mathbf{a}^{(1)}} K_{\mathbf{a}}(\mathbf{a})^{(1)^{-1}}-\mathbf{a}\right) \sqrt{L_{\mathbf{a}}}
$$

Then for $m>0$ we have

$$
\begin{aligned}
{\left[B_{\mathbf{a}} \bar{\gamma}^{m} \mathbf{p}, B_{\mathbf{a}} \mathbf{q}\right]=\left[B_{\mathbf{a}^{(1)}}\right.} & \left.\bar{\gamma}^{m-1} \mathbf{p}, K_{\mathbf{a}^{(1)}} K_{\mathbf{a}}(\mathbf{a})^{(1)^{-1}} \sqrt{L_{\mathbf{a}}} \mathbf{q}\right] \\
& =\left[B_{\mathbf{a}^{(1)}}\left(\mathbf{a}^{(1)}\right)^{(m-1)} \mathbf{a}^{(1)^{[m-1]}} \mathbf{p}, K_{\mathbf{a}}(\mathbf{a})^{(1)^{-1}} \sqrt{L_{\mathbf{a}}} \mathbf{q}\right]=0 .
\end{aligned}
$$

Analogously,

$$
\begin{aligned}
{\left[B_{\mathbf{a}} \mathbf{p}, B_{\mathbf{a}} \mathbf{q}\right]=\left[K_{\mathbf{a}^{(1)}} K_{\mathbf{a}}(\mathbf{a})^{(1)^{-1}}\right.} & \left.\sqrt{L_{\mathbf{a}}} \mathbf{p}, K_{\mathbf{a}^{(1)}} K_{\mathbf{a}}(\mathbf{a})^{(1)^{-1}} \sqrt{L_{\mathbf{a}}} \mathbf{q}\right]+\left[\overline{\mathbf{a}} \mathbf{a} L_{\mathbf{a}} \mathbf{p}, \mathbf{q}\right] \\
= & {\left[\left(L_{\mathbf{a}} K_{\mathbf{a}}(\mathbf{a})^{(1)^{-1}}+1-K_{\mathbf{a}}(\mathbf{a})^{-1}\right) \mathbf{p}, \mathbf{q}\right]=[\mathbf{p}, \mathbf{q}] . }
\end{aligned}
$$


As a corollary of Lemma 5.2 and Proposition 6.4 we obtain that an element $F \in \mathbf{H}_{2}(\mathcal{T})$ vanishes at the point $\mathbf{c} \in \mathbb{D}(\mathcal{T})$ if and only if it can be written as $F=B_{\mathbf{c}} G$ where $G \in \mathbf{H}_{2}(\mathcal{T})$ is such that $[G, G]=[F, F]$. More generally, one can consider the following homogeneous interpolation problem:

Given $\mathbf{c}_{1}, \ldots, \mathbf{c}_{N} \in \mathbb{D}(\mathcal{T})$ find all $F \in \mathbf{H}_{2}(\mathcal{T})$ such that

$$
F\left(\mathbf{c}_{j}\right)=0, \quad j=1, \ldots, N .
$$

We assume that it is possible to recursively define invertible $\mathbf{k}_{j}$ by

$$
\mathbf{k}_{1}=1, \mathbf{k}_{j+1}=\left(B_{\mathbf{k}_{1}^{(1)} \mathbf{k}_{1}^{-1} \mathbf{c}_{1}} B_{\mathbf{k}_{2}^{(1)} \mathbf{k}_{2}^{-1} \mathbf{c}_{2}} \cdots B_{\mathbf{k}_{j}^{(1)} \mathbf{k}_{j}^{-1} \mathbf{c}_{j}}\right)\left(\mathbf{c}_{j+1}\right) .
$$

Then $F$ is a solution of the interpolation problem (6.3) if and only if

$$
F=\left(B_{\mathbf{k}_{1}^{(1)} \mathbf{k}_{1}^{-1} \mathbf{c}_{1}} B_{\mathbf{k}_{2}^{(1)} \mathbf{k}_{2}^{-1} \mathbf{c}_{2}} \cdots B_{\mathbf{k}_{N}^{(1)} \mathbf{k}_{N}^{-1} \mathbf{c}_{N}}\right) G,
$$

where $G \in \mathbf{H}_{2}(\mathcal{T})$ satisfies $[G, G]=[F, F]$. Indeed, assume that

$$
F=G_{0}=\ldots=B_{\mathbf{k}_{1}^{(1)} \mathbf{k}_{1}^{-1} \mathbf{c}_{1}} B_{\mathbf{k}_{2}^{(1)} \mathbf{k}_{2}^{-1} \mathbf{c}_{2}} \cdots B_{\mathbf{k}_{n}^{(1)} \mathbf{k}_{n}^{-1} \mathbf{c}_{n}} G_{n},
$$

where $G_{n} \in \mathbf{H}_{2}(\mathcal{T})$. Then

$$
0=f\left(\mathbf{c}_{n+1}\right)=\left(\mathbf{k}_{n+1} G_{n}\right)\left(\mathbf{c}_{n+1}\right)=G_{n}\left(\mathbf{k}_{n+1}^{(1)} \mathbf{k}_{n+1}^{-1} \mathbf{c}_{n+1}\right),
$$

and hence

$$
G_{n}=B_{\mathbf{k}_{n+1}^{(1)} \mathbf{k}_{n+1}^{-1} \mathbf{c}_{n+1}} G_{n+1},
$$

where $G_{n+1} \in \mathbf{H}_{2}(\mathcal{T})$, and (6.4) follows by induction.

\section{REFERENCES}

[1] D. Alpay and P. Dewilde. Time-varying signal approximation and estimation. In M. Kaashoek, J.H. van Schuppen, and A.C.M. Ran, editors, Signal processing, scattering and operator theory, and numerical methods (Amsterdam, 1989), volume 5 of Progress in systems and control theory, pages 1-22. Birkhäuser Boston, Boston, MA, 1990.

[2] D. Alpay, P. Dewilde, and H. Dym. Lossless inverse scattering and reproducing kernels for upper triangular operators. In Extension and interpolation of linear operators and matrix functions, pages 61-135. Birkhäuser, Basel, 1990.

[3] D. Alpay and Y. Peretz. Realizations for Schur upper triangular operators. In A. Dijksma, I. Gohberg, M. Kaashoek, and R. Mennicken, editors, Contributions to operator theory in spaces with an indefinite metric, volume 106 of Operator Theory: Advances and Applications, pages 37-90. Birkhäuser Verlag, Basel, 1998.

[4] D. Alpay and D. Volok. Interpolation et espace de Hardy sur l'arbre dyadique: le cas stationnaire. Comptes Rendus Mathématiques, 336:293-298, 2003.

[5] W. Arveson. Interpolation problems in nest algebras. J. Functional Analysis, 20(3):208-233, 1975.

[6] J. Ball, I. Gohberg, and M.A. Kaashoek. Two sided Nudelman interpolation for input-output operators of discrete time-varying systems. Integral Equations Operator Theory, 21:174-211, 1995.

[7] J. Ball and V. Victor. Lax-Phillips scattering and conservative linear systems: a Cuntzalgebra multidimensional setting. Preprint, 2003.

[8] J. Ball and V. Vinnikov. Functional models for representation of the Cuntz algebra. Preprint.

[9] M. Basseville, A. Benveniste, and A. Willsky. Multiscale autoregressive processes. Rapport de Recherche 1206, INRIA, Avril 1990.

[10] M. Basseville, A. Benveniste, and A. Willsky. Multiscale statistical signal processing. In Wavelets and applications (Marseille, 1989), volume 20 of RMA Res. Notes Appl. Math., pages 354-367. Masson, Paris, 1992.

[11] A. Benveniste, R. Nikoukhah, and A. Willsky. Multiscale system theory. Rapport de Recherche 1194, INRIA, Mars 1990. 
[12] A. Benveniste, R. Nikoukhah, and A. Willsky. Multiscale system theory. IEEE Trans. Circuits Systems I Fund. Theory Appl., 41(1):2-15, 1994.

[13] P. Cartier. Géométrie et analyse sur les arbres. In Séminaire Bourbaki, 24ème année (1971/1972), Exp. No. 407, pages 123-140. Lecture Notes in Math., Vol. 317. Springer, Berlin, 1973.

[14] T. Constantinescu and J. L. Johnson. Tensor algebras and displacement structure. I. The Schur algorithm. Z. Anal. Anwendungen, 21(1):3-20, 2002.

[15] T. Constantinescu and J. L. Johnson. Tensor algebras and displacement structure. II. Noncommutative Szegő polynomials. Z. Anal. Anwendungen, 21(3):611-626, 2002.

[16] J. Cuntz. Simple $C^{*}$-algebras generated by isometries. Comm. Math. Phys., 57(2):173-185, 1977.

[17] P. Dewilde and H. Dym. Interpolation for upper triangular operators. In I. Gohberg, editor, Time-variant systems and interpolation, volume 56 of Operator Theory: Advances and Applications, pages 153-260. Birkhäuser Verlag, Basel, 1992.

[18] J. Dixmier. Les $C^{*}$-algèbres et leurs représentations. Deuxième édition. Cahiers Scientifiques, Fasc. XXIX. Gauthier-Villars Éditeur, Paris, 1969.

[19] A. Feintuch and R. Saeks. System theory. A Hilbert space approach., volume 102 of Pure and Applied Mathematics. Academic Press Inc. [Harcourt Brace Jovanovich Publishers], New York, 1982.

[20] I. Kaplansky. Modules over operator algebras. Amer. J. Math., 75:839-858, 1953.

[21] R. M. Loynes. Linear operators in VH-spaces. Trans. Amer. Math. Soc., 116:167-180, 1965.

[22] O.Bratteli and P. Jorgensen. Wavelets through a looking glass. Applied and Numerical Harmonic Analysis. Birkhäuser Boston Inc., Boston, MA, 2002. The world of the spectrum.

[23] W. Paschke. Inner product spaces over $B^{*}$-algebras. Trans. Amer. Math. Soc., 1982:443-468, 1973.

[24] J.P. Serre. Arbres, amalgames, $\mathrm{SL}_{2}$. Société Mathématique de France, Paris, 1977. Avec un sommaire anglais, Rédigé avec la collaboration de Hyman Bass, Astérisque, No. 46.

Department of Mathematics, Ben-Gurion University of the Negev, Beer-Sheva 84105, ISRAEL

E-mail address: dany@math.bgu.ac.il

Department of Mathematics, Ben-Gurion University of the Negev, Beer-Sheva 84105, ISRAEL

E-mail address: volok@math.bgu.ac.il 